\title{
GENERALIZED EXTREME SHOCK MODELS WITH A POSSIBLY INCREASING THRESHOLD
}

\author{
Pasouale Cirillo and Jürg Hüsler \\ Institute of Mathematical Statistics and Actuarial Sciences, \\ University of Bern \\ CH-3012, Bern, Switzerland \\ Email: pasquale.cirillo@stat.unibe.ch
}

\begin{abstract}
We propose a generalized extreme shock model with a possibly increasing failure threshold. Although standard models assume that the crucial threshold for the system might only decrease over time, because of weakening shocks and obsolescence, we assume that, especially at the beginning of the system's life, some strengthening shocks might increase the system tolerance to large shock. This is, for example, the case of turbines' running-in in the field of engineering. On the basis of parametric assumptions, we provide theoretical results and derive some exact and asymptotic univariate and multivariate distributions for the model. In the last part of the article we show how to link this new model to some nonparametric approaches proposed in the literature.
\end{abstract}

\section{INTRODUCTION}

The setup in extreme shock models is a family $\left\{\left(X_{k}, Y_{k}\right), k \geq 0\right\}$ of independent identically distributed (i.i.d.) two-dimensional random vectors, with $X_{k}$ the magnitude of the $k$ th shock and $Y_{k}$ the time between the $(k-1)$ st and the $k$ th shock. The main object of interest is the lifetime/failure time of the system by assuming certain schemes for the failure. These models are motivated by the possible breakdown of a material or of a system subject to random shocks of random magnitude, as it occurs in engineering. It is easy to see useful applications also in other fields, such as economics, medicine, and biology.

Cumulative shock models and extreme shock models are discussed, as well as mixtures of both models, in Gut and Hüsler [8,9] and the references therein. 
In the cumulative shock model, we consider

$$
T_{n}=\sum_{k \leq n} Y_{k} \quad \text { and } \quad S_{n}=\sum_{k \leq n} X_{k}
$$

for $n \geq 1$, with $T_{0}=S_{0}=0$. The failure of the system occurs if $S_{n}>\alpha$ for some $n$ and $\alpha$. Here, $\alpha$ denotes the critical threshold of the system. The time until the system fails the first time or the failure time $T_{\tau}$ with $\tau=\min \left\{n: S_{n}>\alpha\right\}$ are then of interest. For results, see Gut [6].

In the simple extreme shock model, one large or extreme shock, larger than a given failure (or crucial) threshold $\gamma$, might cause the default of the system. The lifetime of the system is, in this case, defined as $T_{v}$, where

$$
v=\min \left\{n: X_{n}>\gamma\right\}
$$

This model was dealt with in Gut and Hüsler [8], and Gut [7].

Gut and Hüsler [9] extended this simple model to a more realistic framework by assuming that the failure threshold is not constant but that it might vary with time, depending on the experienced shocks. In detail, they assume that a large but not fatal shock might effect the system's tolerance to subsequent shocks, because of cracks in the structure, for example. To be more exact, for a fixed $\alpha_{0}>0$ a shock $X_{i}$ can damage the system if it is larger than a certain boundary value $\beta<\alpha_{0}{ }^{1}$ As long as $X_{i}<\alpha_{0}$, the system does not fail. The crucial hypothesis is the following: If a first nonfatal shock comes with values in $\left[\beta, \alpha_{0}\right]$, the maximum load limit of the system is no longer $\alpha_{0}$ but decreases to $\alpha_{1} \in\left[\beta, \alpha_{1}\right]$. At this point, if another large but not too strong shock occurs in $\left[\beta, \alpha_{0}\right]$, the new crucial threshold is lowered again to $\alpha_{2} \in\left[\beta, \alpha_{1}\right]$ and so on until the system fails. We could call all this "the risky threshold mechanism." Naturally, for $\forall t$

$$
\alpha_{0} \geq \alpha_{1} \geq \alpha_{2} \geq \cdots \geq \beta
$$

Hence, one can define the stopping time $v=\min \left\{n: X_{n} \geq \alpha_{L(n-1)}\right\}$ with $L(n)=$ $\sum_{i=1}^{n} 1_{\left\{X_{i} \geq \beta\right\}}$ and $L(0)=0$. Gut and Hüsler [9] have shown that the results for generalized extreme shock models (GESMs) are identical to the simple extreme case for nonrandom $\alpha_{k}$, whereas this is not true in the random case. We refer to the original article for more details.

Even if the modeling of GESMs is surely sensible, sometimes it can be worth considering a default threshold $\alpha$ that might even increase, at least initially, say in particular for a running-in period of some equipment. This is, for example, the case of turbines' breaking-in in the field of engineering, but other applications can be found in electric networks and biology (e.g., Siphonophora in their growing process, Dunn, Pugh, and Haddock [3]). We present in the following section this more general model with some theoretical results, which are based on parametric assumptions.

\footnotetext{
${ }^{1}$ The value $\beta$ can also vary over time. The only requirement is that always be lower than the corresponding failure threshold.
} 
In Section 3 we derive some exact and asymptotic univariate and multivariate distributions of the parametric model.

Generalized extreme shock models can even be studied using nonparametric techniques. In Cirillo and Hüsler [1] a nonparametric urn-based approach to extreme shock models is proposed. In Section 4 we briefly show how the same approach could be used to model the increasing threshold. Section 5 concludes the article.

\section{EXTREME SHOCK MODELS WITH A POSSIBLE INCREASING THRESHOLD}

In some applications, a system has a running-in period during which the critical load can increase and the structure is strengthened because, at the beginning, the loads or shocks are large but nonfatal. This might happen, in particular, until the first damage or crack. After such an event, the system can only be weakened. Such a pattern can be modeled as follows.

We let the arrival times $T_{i}$ of the shocks $X_{i}$ be, as mentioned, a partial sum of i.i.d. interarrival times $Y_{j}$ with distribution $G$. The loads $X_{i}, i \geq 1$, are an i.i.d. sequence of random variables (r.v.s) with distribution $F$. A shock or stroke $X_{i}$ is strengthening the material if $X_{i} \in[\gamma, \beta)$. At the beginning, the material supports a maximal load $\alpha$, the critical threshold. After a strengthening stroke, the maximal load becomes larger, say $\alpha_{1}=\alpha+b_{1}$ with $b_{1}>0$. This boundary increases with each strengthening stroke, inducing boundaries $\alpha_{j}=\alpha+b_{j}, j \geq 1$ with $b_{j} \uparrow$. After the first harmful stroke larger than $\beta$, but smaller than the critical level at this time point, the load boundary decreases because of possible cracks or some weakening of the material. If it has reached the level $\alpha_{k}$ (because of $k$ strengthening strokes before the first harmful stroke), the critical level now becomes $\alpha_{k}-c_{1}$ and decreases further by the next harmful, nonfatal strokes to $\alpha_{k}-c_{2}, \alpha_{k}-c_{3}, \ldots$, with $c_{j} \uparrow(\geq 0)$. This is shown in Figure 1. There might be an upper load limit $\alpha^{*}$ for the $\alpha_{k}$, as well a lower load limit $\alpha_{*}$ for the $\alpha_{k}-c_{l}$. We set $\alpha_{*} \geq \beta$. It is convenient to set $b_{0}=c_{0}=0$. For notational reasons we define the

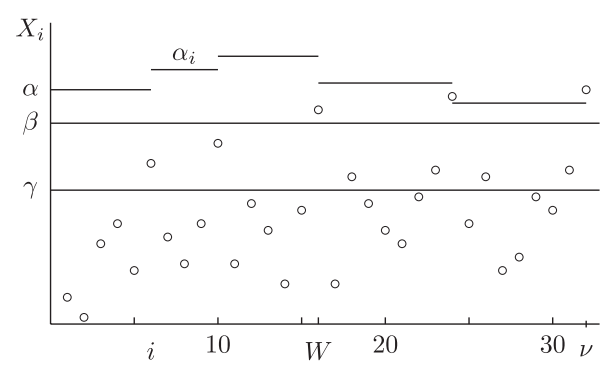

FIGURE 1. Realization of a sequence of shocks with strengthening and weakening load limits, depending on the values $X_{i}$. Here, we have $v=32, W=16, N_{+}(v)=2$, and $N_{-}(v)=2$. 
number $N_{-}(n)$ of weakening shocks $X_{i}, i<n$, before the $n$th shock:

$$
N_{-}(n)=\sum_{i<n} 1\left(X_{i} \in\left[\beta, \alpha+b_{N_{+}(i)}-c_{N_{-}(i)}\right)\right.
$$

with $N_{-}(0)=0$; and define the number $N_{+}(n)$ of strengthening strokes $X_{i}, i<n$, before the $n$-th shock and before the first weakening or fatal shock:

$$
N_{+}(n)=\sum_{i<n} 1\left(X_{i} \in[\gamma, \beta), N_{-}(i)=0\right)
$$

with $N_{+}(0)=0$. Note that the critical boundary for $X_{i}$ is $\alpha_{i}=\alpha+b_{N_{+}(i)}-c_{N_{-}(i)}$.

Additionally let $W$ be the index of the first harmful shock larger than $\beta$. It could indicate even a fatal shock being larger than the critical boundary at this time point. Hence, if such a shock occurs,

$$
W=\inf \left\{i: X_{i} \geq \beta\right\} \leq \infty
$$

If the set is empty, we set $W=\infty$. Hence, the shock $X_{i}$ has no impact if $X_{i} \leq \gamma$; it induces a strengthening of the material if $i<W$ and $X_{i} \in[\gamma, \beta)$ and it is fatal if $X_{i} \geq \alpha_{i}=\alpha+b_{N_{+}(i)}-c_{N_{-}(i)}$. Note, also, that $N_{+}(k)=N_{+}(W)$ for all $k \geq W(<\infty)$.

First, we analyze in Section 3 the distribution of the number $v$ of shocks until the first fatal shock:

$$
v=\min \left\{i: X_{i} \geq \alpha+b_{N_{+}(i)}-c_{N_{-}(i)}\right\},
$$

where we use $b_{0}=0$ and $c_{0}=0$. The time until the fatal shock is thus $T_{v}$. Its distribution depends on the distribution of $v$ and $G$.

We consider the asymptotic behavior of these random variables by letting the parameters $\alpha=\alpha(t), \beta=\beta(t)$, and $\gamma=\gamma(t)$ tend to $x_{F} \leq \infty$ as $t \rightarrow \infty$, where $x_{F}$ denotes the upper end point of the distribution $F$. We assume that $x_{F}$ is a continuity point of $F$. For the limit distributions, certain additional restrictions will be imposed also on the $b_{k}$ and $c_{k}$, being also dependent on $t$. Hence, $v=v(t)$ will tend to $\infty$, in general, depending on the underlying distribution $F$.

\section{THE DISTRIBUTION OF $v$}

The distribution of $v$ can be derived in this more general model as in the basic generalized extreme shock model of Gut and Hüsler (2005). First we derive the exact distribution and then analyze the asymptotic distributions which depend on the behaviour of the sequences $b_{k}$ and $c_{k}$. For the derivation we use the notation

$$
\alpha_{k, l}=\alpha+b_{k}-c_{l}
$$

for any $k, l \geq 0$. 


\subsection{The Exact Distribution}

To derive $P\{v>m\}$, we have to condition on the other random variables. If $N_{-}$ $(m+1)=0$, then $W>m$ and, simply, $P\left\{v>m, N_{-}(m+1)=0\right\}=F^{m}(\beta)$. If $l>0$ with $k<j$ and $m \geq j+l-1$, we consider the joint distribution

$$
\begin{aligned}
P\{v & \left.>m, N_{+}(m)=k, N_{-}(m+1)=l, W=j\right\} \\
= & \left(\begin{array}{c}
j-1 \\
k
\end{array}\right) F^{j-1-k}(\gamma)[\bar{F}(\gamma)-\bar{F}(\beta)]^{k} \\
& \times\left(\begin{array}{c}
m-j \\
l-1
\end{array}\right) F^{m-j-l+1}(\beta) \prod_{h=1}^{l}\left[\bar{F}(\beta)-\bar{F}\left(\alpha_{k, h-1}\right)\right]
\end{aligned}
$$

or, for $m \geq j+l$ and $k<j$,

$$
\begin{aligned}
P\{v= & \left.m, N_{+}(m)=k, N_{-}(m)=l, W=j\right\} \\
= & \left(\begin{array}{c}
j-1 \\
k
\end{array}\right) F^{j-1-k}(\gamma)[\bar{F}(\gamma)-\bar{F}(\beta)]^{k} \\
& \times\left(\begin{array}{c}
m-j-1 \\
l-1
\end{array}\right) F^{m-j-l}(\beta) \prod_{h=1}^{l}\left[\bar{F}(\beta)-\bar{F}\left(\alpha_{k, h-1}\right)\right] \bar{F}\left(\alpha_{k, l}\right) .
\end{aligned}
$$

If $k \geq j$ or $m \leq j+l-1$, the latter probabilities are zero.

By summing the appropriate terms, we get the exact univariate and multivariate distributions for $v, N_{+}(m), N_{-}(l)$, and $W$ as well as for $N_{+}(v)$ and $N_{-}(v)$. For instance, the joint distribution of $N_{+}(v), N_{-}(v)$ with $l \geq 1$ is

$$
\begin{aligned}
P\left\{N_{+}(v)=k, N_{-}(v)=l\right\} & \\
= & \sum_{m} P\left\{v=m, N_{+}(m)=k, N_{-}(m)=l\right\} \\
= & \sum_{m, j}\left(\begin{array}{c}
j-1 \\
k
\end{array}\right)\left(\begin{array}{c}
m-j-1 \\
l-1
\end{array}\right) F^{j-k-1}(\gamma)[\bar{F}(\gamma)-\bar{F}(\beta)]^{k} F^{m-j-l}(\beta) \\
& \times \prod_{h=0}^{l-1}\left\{1-\bar{F}\left(\alpha_{k, h}\right) / \bar{F}(\beta)\right\} \bar{F}^{l}(\beta) \bar{F}\left(\alpha_{k, l}\right) \\
= & \sum_{j \geq k+1}\left(\begin{array}{c}
j-1 \\
k
\end{array}\right) F^{j-k-1}(\gamma) \sum_{m \geq j+l}\left(\begin{array}{c}
m-j-1 \\
l-1
\end{array}\right) F^{m-j-l}(\beta) \bar{F}^{l}(\beta) \\
& \times[\bar{F}(\gamma)-\bar{F}(\beta)]^{k} \prod_{h=0}^{l-1}\left\{1-\bar{F}\left(\alpha_{k, h}\right) / \bar{F}(\beta)\right\} \bar{F}\left(\alpha_{k, l}\right) .
\end{aligned}
$$


Note that the sums are summing all of the probabilities of a negative binomial distribution, hence,

$$
\begin{aligned}
P\left\{N_{+}(v)=k, N_{-}(v)=l\right\} & \\
= & \sum_{h \geq 0}\left(\begin{array}{c}
h+k \\
k
\end{array}\right) F^{h}(\gamma) \\
& \times[\bar{F}(\gamma)-\bar{F}(\beta)]^{k} \prod_{h=0}^{l-1}\left\{1-\bar{F}\left(\alpha_{k, h}\right) / \bar{F}(\beta)\right\} \bar{F}\left(\alpha_{k, l}\right) \\
= & \bar{F}^{-k-1}(\gamma)[\bar{F}(\gamma)-\bar{F}(\beta)]^{k} \prod_{h=0}^{l-1}\left\{1-\bar{F}\left(\alpha_{k, h}\right) / \bar{F}(\beta)\right\} \bar{F}\left(\alpha_{k, l}\right) \\
= & {[1-\bar{F}(\beta) / \bar{F}(\gamma)]^{k} \prod_{h=0}^{l-1}\left\{1-\bar{F}\left(\alpha_{k, h}\right) / \bar{F}(\beta)\right\}\left[\bar{F}\left(\alpha_{k, l}\right) / \bar{F}(\gamma)\right] . }
\end{aligned}
$$

For the case $l=0$, we obtain in the same way

$$
\begin{aligned}
& P\left\{N_{+}(v)=k, N_{-}(v)=0\right\} \\
& \quad=\sum_{m>k} P\left\{v=m, N_{+}(m)=k, N_{-}(m)=0, W=m\right\} \\
& \quad=\sum_{m>k}\left(\begin{array}{c}
m-1 \\
k
\end{array}\right) F^{m-k-1}(\gamma)[\bar{F}(\gamma)-\bar{F}(\beta)]^{k} \bar{F}\left(\alpha_{k, 0}\right) \\
& =[1-\bar{F}(\beta) / \bar{F}(\gamma)]^{k}\left[\bar{F}\left(\alpha_{k, 0}\right) / \bar{F}(\gamma)\right] .
\end{aligned}
$$

Other exact distributions can be derived in the same way by appropriate summation. Sometimes, for the derivation of the asymptotic distributions, we have to approximate these sums to simplify the formulas.

We give, for later use, the following exact distribution:

$$
\begin{aligned}
P\{v> & \left.m, N_{-}(v)>0\right\} \\
= & \sum_{k \geq 0, l>0, j \geq 1} P\left\{v>m, N_{+}(m)=k, N_{-}(m+1)=l, W=j\right\} \\
= & \sum_{k \geq 0, l>0, j \geq 1}\left(\begin{array}{c}
j-1 \\
k
\end{array}\right) F^{j-1-k}(\gamma)[\bar{F}(\gamma)-\bar{F}(\beta)]^{k} \\
& \times\left(\begin{array}{c}
m-j \\
l-1
\end{array}\right) F^{m-j-l+1}(\beta) \prod_{h=1}^{l}\left[\bar{F}(\beta)-\bar{F}\left(\alpha_{k, h-1}\right)\right] .
\end{aligned}
$$




\subsection{The Asymptotic Distribution}

For the asymptotic behavior, let $\alpha(t) \rightarrow \infty, \beta(t) \rightarrow \infty$, and $\gamma(t) \rightarrow \infty$ as $t \rightarrow \infty$. The asymptotic behavior depends also on the assumptions of the sequences $b_{k}$ and $c_{k}$, which might depend also on the parameter $t$. However, we do not indicate the dependence on the parameter $t$. From the above finite distributions, it is reasonable to use the conditions $\bar{F}(\beta(t)) \rightarrow 0$ as $t \rightarrow \infty$ :

$$
\lim _{t} \frac{\bar{F}(\beta(t))}{\bar{F}(\gamma(t))}=g \in[0,1]
$$

and

$$
\lim _{t} \frac{\bar{F}\left(\alpha(t)+b_{k}(t)-c_{l}(t)\right)}{\bar{F}(\beta(t))}=\lim _{t} \frac{\bar{F}\left(\alpha_{k, l}(t)\right)}{\bar{F}(\beta(t))}=a_{k, l} \in[0,1] .
$$

Obviously, the $a_{k, l}$ are monotone by the assumed monotonicity of the sequences $b_{k}$ and $c_{k}$ (i.e., monotone decreasing in $k$ with $l$ fixed, and monotone increasing in $l$ with $k$ fixed). We consider only the interesting cases with $g, a_{k, l} \in(0,1)$. The simplest case occurs if $a_{k, l}=a \in(0,1)$ for all $k$ and $l$. This implies that

$$
\prod_{h=1}^{l}\left[\bar{F}(\beta)-\bar{F}\left(\alpha+b_{k}-c_{h-1}\right)\right] \sim[\bar{F}(\beta)(1-a)]^{l}
$$

for each $l \geq 1$. However, in general, we approximate

$$
\prod_{h=1}^{l}\left[\bar{F}(\beta)-\bar{F}\left(\alpha+b_{k}-c_{h-1}\right)\right] \sim \bar{F}^{l}(\beta) \prod_{h=1}^{l}\left(1-a_{k, h}\right)
$$

for each $l \geq 1$. We use for notational reason also $\prod_{h=1}^{0}\left(1-a_{k, h}\right)=1$. To simplify the notation, we do not indicate the dependence on $t$ in the following (e.g., we write $v$ instead of $v(t))$.

THEOREM 1: If (9) and (10) hold with $g, a_{k, l} \in[0,1]$, then for any $k \geq 0, l \geq 0$

$$
P\left\{N_{+}(v)=k, N_{-}(v)=l\right\} \rightarrow g(1-g)^{k} \prod_{h=0}^{l-1}\left(1-a_{k, h}\right) a_{k, l}
$$

as $t \rightarrow \infty$.

Proof: Use (6) for $l \geq 1$ and (7) for $l=0$, with assumptions (9) and (10) to immediately derive the claim, as $t \rightarrow \infty$. 
For the particular simple case that $a_{k, h}=a$ for all $k$ and $h$, this limit distribution is the product of two geometric distributions:

$$
P\left\{N_{+}(v)=k, N_{-}(v)=l\right\} \rightarrow g(1-g)^{k}(1-a)^{l} a .
$$

Because of the particular assumptions, the number of strengthening strokes does not have an influence on the number of weakening strokes asymptotically, which shows the asymptotic independence of $N_{+}(v)$ and $N_{-}(v)$ in this case.

THEOREM 2: If (9) and (10) hold with $a_{k, l} \in(0,1]$ for each $k$ and $l$, we have the following:

(i) For $k \geq 0=l$,

$$
\lim _{t \rightarrow \infty} P\left\{v \geq z / \bar{F}(\beta), N_{+}(v)=k, N_{-}(v)=0\right\}=\int_{z / g}^{\infty} v^{k} e^{-v} d v g(1-g)^{k} a_{k, 0} / k ! .
$$

(ii) For $l \geq 1$ and $k \geq 0$,

$$
\begin{aligned}
\lim _{t \rightarrow \infty} & P\left\{v \geq z / \bar{F}(\beta), N_{+}(v)=k, N_{-}(v)=l\right\} \\
= & \int_{z}^{\infty} \int_{0}^{1} y^{k}(1-y)^{l-1} \exp \left\{-y u\left(g^{-1}-1\right)\right\} d y \exp \{-u\} u^{k+l} d u \\
& \times((1-g) / g)^{k} \prod_{h=0}^{l-1}\left(1-a_{k, h}\right) a_{k, l} /(k !(l-1) !)
\end{aligned}
$$

as $t \rightarrow \infty$.

PROOF: Let $k \geq 0=l$ and $z_{\beta}=z / \bar{F}(\beta)$.

$$
\begin{aligned}
P\{v \geq & \left.z_{\beta}, N_{+}(v)=k, N_{-}(v)=0\right\}= \\
& =\sum_{m \geq z_{\beta}} P\left\{v=m, N_{+}(m)=k, N_{-}(m)=0\right\} \\
& =\sum_{m=z_{\beta}}^{\infty}\left(\begin{array}{c}
m-1 \\
k
\end{array}\right) F^{m-k-1}(\gamma)[\bar{F}(\gamma)-\bar{F}(\beta)]^{k} \bar{F}\left(\alpha_{k, 0}\right) \\
& \sim \sum_{m=z_{\beta}}^{\infty} \frac{m^{k}}{k !} F^{m}(\gamma)[\bar{F}(\gamma)-\bar{F}(\beta)]^{k} \bar{F}\left(\alpha_{k, 0}\right) \\
& \sim \frac{1}{k !} \int_{z}^{\infty} v^{k} \exp \{-v \bar{F}(\gamma) / \bar{F}(\beta)\} d v(\bar{F}(\beta))^{-k-1}[\bar{F}(\gamma)-\bar{F}(\beta)]^{k} \bar{F}\left(\alpha_{k, 0}\right) \\
& \rightarrow \frac{1}{k !} \int_{z / g}^{\infty} y^{k} e^{-y} d y(1-g)^{k} g a_{k, 0}
\end{aligned}
$$

as $t \rightarrow \infty$. If $k=0$, then the integral is simply $e^{-z / g}$. 
Now, let $l>0$. By (4) we have

$$
\begin{aligned}
P\left\{v \geq z_{\beta}, N_{+}(v)=k, N_{-}(v)=l\right\} & \\
= & \sum_{m \geq z \beta} P\left\{v=m, N_{+}(m)=k, N_{-}(m)=l\right\} \\
= & \sum_{m=z_{\beta}}^{\infty} \sum_{j=k+1}^{m-l}\left(\begin{array}{c}
j-1 \\
k
\end{array}\right)\left(\begin{array}{c}
m-j-1 \\
l-1
\end{array}\right) F^{j-k-1}(\gamma) F^{m-j-l}(\beta) \\
& \quad \times \bar{F}^{k}(\gamma)(1-g+o(1))^{k} \bar{F}^{l+1}(\beta) \prod_{h=0}^{l-1}\left(1-a_{k, h}+o(1)\right)\left(a_{k, l}+o(1)\right)
\end{aligned}
$$

Split the inner sum into three parts, with $j \leq \epsilon m, \epsilon m<j<(1-\epsilon) m$, and $j \geq$ $(1-\epsilon) m$, where we used $W=j$ in the summands. The first and the third sum are asymptotically negligible as $\epsilon \rightarrow 0$. This can be shown through derivations similar to the following ones for the second sum. In fact, for the second sum, we have

$$
\begin{aligned}
P\{v \geq & \left.z_{\beta}, N_{+}(v)=k, N_{-}(v)=l, \epsilon m<W<(1-\epsilon) m\right\} \\
= & \sum_{m=z_{\beta}}^{\infty} \sum_{j>\epsilon m}^{(1-\epsilon) m}\left(\begin{array}{c}
j-1 \\
k
\end{array}\right)\left(\begin{array}{c}
m-j-1 \\
l-1
\end{array}\right) F^{j-k-1}(\gamma) F^{m-j-l}(\beta) \\
& \times \bar{F}^{k}(\gamma) \bar{F}^{l+1}(\beta)(1-g+o(1))^{k} \prod_{h=0}^{l-1}\left(1-a_{k, h}+o(1)\right)\left(a_{k, l}+o(1)\right) \\
\sim & \sum_{m=z_{\beta}}^{\infty} \sum_{j>\epsilon m}^{[(1-\epsilon) m]} \frac{(j-1) !(m-j-1) !}{k !(j-1-k) !(l-1) !(m-j-l) !} F^{j-k-1}(\gamma) F^{m-j-l}(\beta) \\
& \times \bar{F}^{k}(\gamma) \bar{F}^{l+1}(\beta)(1-g)^{k} \prod_{h=0}^{l-1}\left(1-a_{k, h}\right) a_{k, l} \\
& \sum_{m=z_{\beta}}^{\infty} \sum_{j>\epsilon m}^{[(1-\epsilon) m]} \frac{j^{k}(m-j)^{l-1}}{k !(l-1) !} F^{j-k-1}(\gamma) F^{m-j-l}(\beta) \\
& \times \bar{F}^{k}(\gamma) \bar{F}^{l+1}(\beta)(1-g)^{k} \prod_{h=0}^{l-1}\left(1-a_{k, h}\right) a_{k, l}=: P_{t} .
\end{aligned}
$$

Now, we approximate the sums by integrals:

$$
\begin{aligned}
P_{t} \sim & \sum_{m=z_{\beta}}^{\infty} \int_{\epsilon}^{1-\epsilon}(y m)^{k}(m(1-y))^{l-1} F^{y m}(\gamma) F^{m(1-y)}(\beta) d y(m / k !(l-1) !) \\
& \times \bar{F}^{k}(\gamma) \bar{F}^{l+1}(\beta)(1-g)^{k} \prod_{h=0}^{l-1}\left(1-a_{k, h}\right) a_{k, l}
\end{aligned}
$$




$$
\begin{aligned}
= & \sum_{m=z_{\beta}}^{\infty} \int_{\epsilon}^{1-\epsilon} y^{k}(1-y)^{l-1} F^{y m}(\gamma) F^{m(1-y)}(\beta) d y m^{k+l} \\
& \times \bar{F}^{k}(\gamma) \bar{F}^{l+1}(\beta)(1-g)^{k} \prod_{h=0}^{l-1}\left(1-a_{k, h}\right) a_{k, l} /(k !(l-1) !) \\
\sim & \int_{z}^{\infty} \int_{\epsilon}^{1-\epsilon} y^{k}(1-y)^{l-1} F^{y u / \bar{F}(\beta)}(\gamma) F^{(1-y) u / \bar{F}(\beta)}(\beta) d y u^{k+l} d u \\
& \times \bar{F}^{k}(\gamma) \bar{F}^{-k}(\beta)(1-g)^{k} \prod_{h=0}^{l-1}\left(1-a_{k, h}\right) a_{k, l} /(k !(l-1) !) \\
\sim & \int_{z}^{\infty} \int_{\epsilon}^{1-\epsilon} y^{k}(1-y)^{l-1} F^{y u / \bar{F}(\beta)}(\gamma) F^{(1-y) u / \bar{F}(\beta)}(\beta) d y u^{k+l} d u \\
& \times g^{-k}(1-g)^{k} \prod_{h=0}^{l-1}\left(1-a_{k, h}\right) a_{k, l} /(k !(l-1) !) .
\end{aligned}
$$

The factor $F^{y u / \bar{F}(\beta)}(\gamma) F^{(1-y) u / \bar{F}(\beta)}(\beta)$ can be approximated for large $t$ by $\exp (-(1+o(1)) y u \bar{F}(\gamma) / \bar{F}(\beta)-(1+o(1))(1-y) u) \sim \exp \left(-\left[y u\left(g^{-1}-1\right)-u\right]\right)$

uniformly for $u$ bounded. Hence, by the dominated convergence, we get

$$
\begin{aligned}
P_{t} \sim & \int_{z}^{\infty} \int_{\epsilon}^{1-\epsilon} y^{k}(1-y)^{l-1} \exp \left\{-y u\left(g^{-1}-1\right)\right\} d y \exp \{-u\} u^{k+l} d u \\
& \times((1-g) / g)^{k} \prod_{h=0}^{l-1}\left(1-a_{k, h}\right) a_{k, l} /(k !(l-1) !) \\
& \rightarrow \int_{z}^{\infty} \int_{0}^{1} y^{k}(1-y)^{l-1} \exp \left\{-y u\left(g^{-1}-1\right)\right\} d y \exp \{-u\} u^{k+l} d u \\
& \times((1-g) / g)^{k} \prod_{h=0}^{l-1}\left(1-a_{k, h}\right) a_{k, l} /(k !(l-1) !)
\end{aligned}
$$

as $\epsilon \rightarrow 0$.

If we set $z=0$, the integrals of both statements can be determined explicitly, which implies the result of Theorem 1 in both cases $l=0$ and $l>0$.

Other limit distributions are determined by summing the terms $P\{v=m$, $\left.N_{+}(m)=k, N_{-}(v)=l, W=j\right\}$. Only under additional assumptions can these distributions be simplified. Let us deal with such a particular case that generalizes Theorem 5.1 in Gut and Hüsler [9]. The formulas and the sums or integrals can be simplified, for instance, if the impact of the strengthening strokes is asymptotically negligible 
(i.e., when $a_{k, h}=a_{h}$ for all $k$ and $h$ ). In this case, we consider the limit distribution of $v$ and $T_{\nu}$.

THEOREM 3: If (9) and (10) hold with $a_{k, h}=a_{h} \in[0,1]$ for each $k$ and $h \geq 1$, then for $z_{\beta}=z / \bar{F}(\beta)$ with $z>0$,

$$
\begin{aligned}
P\left\{v>z_{\beta}\right\} & \rightarrow \sum_{l=0}^{\infty} \frac{z^{l}}{l !} e^{-z} \prod_{h=0}^{l-1}\left(1-a_{h}\right) \\
& =1-H(z)
\end{aligned}
$$

and

$$
P\left\{T_{v}>z_{\beta}\right\} \rightarrow 1-H(z / \mu)
$$

as $t \rightarrow \infty$, where $\mu=E\left(Y_{1}\right)<\infty$.

ProOF: The assumptions imply that $\prod_{h=0}^{l-1}\left(1-a_{k, h}\right)=\prod_{h=0}^{l-1}\left(1-a_{h}\right)=: \tilde{a}_{l}$. Let $\tilde{a}_{0}=1$.

(i) For the first statement, we start with (8) and apply assumptions (9) and (10) to derive the limiting distribution:

$$
\begin{aligned}
P\{v> & \left.m, N_{-}(m)>0\right\} \\
= & \sum_{j=1}^{m} \sum_{l=1}^{m-j+1} \sum_{k=0}^{j-1}\left(\begin{array}{c}
j-1 \\
k
\end{array}\right) F^{j-1-k}(\gamma)[\bar{F}(\gamma)-\bar{F}(\beta)]^{k} \\
& \times\left(\begin{array}{c}
m-j \\
l-1
\end{array}\right) F^{m-j-l+1}(\beta) \prod_{h=1}^{l}\left[\bar{F}(\beta)-\bar{F}\left(\alpha_{k, h-1}\right)\right] \\
\sim & \sum_{j=1}^{m} \sum_{l=1}^{m-j+1} \sum_{k=0}^{j-1}\left(\begin{array}{c}
j-1 \\
k
\end{array}\right) F^{j-1-k}(\gamma)[\bar{F}(\gamma)-\bar{F}(\beta)]^{k} \\
& \times\left(\begin{array}{c}
m-j \\
l-1
\end{array}\right) F^{m-j-l+1}(\beta) \bar{F}^{l}(\beta) \tilde{a}_{l} .
\end{aligned}
$$

Now, using that the sum on $k$ can be simplified because it is a binomial sum, we get for this sum,

$$
\begin{aligned}
(F(\gamma) & +\bar{F}(\gamma)-\bar{F}(\beta))^{j-1}=(F(\beta))^{j-1} \\
& =\sum_{j=1}^{m} \sum_{l=1}^{m-j+1} F^{j-1}(\beta)\left(\begin{array}{c}
m-j \\
l-1
\end{array}\right) F^{m-j-l+1}(\beta) \bar{F}^{l}(\beta) \tilde{a}_{l} \\
& =\sum_{l=1}^{m} \sum_{j=1}^{m-l+1}\left(\begin{array}{c}
m-j \\
l-1
\end{array}\right) F^{m-l}(\beta) \bar{F}^{l}(\beta) \tilde{a}_{l}
\end{aligned}
$$




$$
\begin{aligned}
& =\sum_{l=1}^{m}\left(\begin{array}{c}
m \\
l
\end{array}\right) F^{m-l}(\beta) \bar{F}^{l}(\beta) \tilde{a}_{l} \\
& \rightarrow \sum_{l=1}^{\infty} \frac{z^{l}}{l !} \exp (-z) \tilde{a}_{l}
\end{aligned}
$$

using the normalization $m=z / \bar{F}(\beta)$, which tends to $\infty$.

(ii) The second statement is immediate by applying the weak law of large numbers for $T_{v} / \nu \stackrel{p}{\rightarrow} \mu$, as $t \rightarrow \infty$.

In other more general cases we have to sum the terms of Theorem 2 to get the limit distribution of $v$ and $T_{v}$. Notice also that the dependence between $X_{k}$ and $Y_{k}$ has no influence on the limit distribution of $T_{v}$.

\section{A LINKTO URN-BASED SHOCK MODELS}

A nonparametric approach to shock models has been recently proposed in Cirillo and Hüsler [1,2]. These nonparametric models are based on combinatorial processes and, in particular, on combinations of Polya-like urn schemes. Extreme shock models are modeled in Cirillo and Hüsler [2] using a special version of the reinforced urn process of Muliere, Secchi, and Walker [10], which allows for a Bayesian nonparametric treatment of shock models.

Generalized extreme shock models are instead modeled in Cirillo and Hüsler [1] by the means of a particular triangular Polya-like urn. This new model is called the urn-based generalized extreme shock model (UbGESM). In the following we aim to show that a similar construction could also be applied, with some modifications, to the increasing threshold shock model we have introduced in Section 2.

For completeness, let us briefly recall the UbGESM. The basic characteristic of the UbGESM construction is to get around the definition of the decreasing threshold mechanism of GESMs, as developed in Gut and Hüsler [9], by creating three different risk areas for the system (no risk or safe, risky, and default), by linking every area to a particular color, and by working with the probability for the process to enter each area. If every time the process enters the risky area the probability of failing increases, this can be obtained with a triangular reinforcement matrix — such a modeling can be considered a sort of intuitive approach to GESMs. In some sense, reinforcing the probability for the system to fail is like making the risky threshold move down and vice versa.

The authors consider an urn containing balls of three different colors: $x$ (safe), $y$ (risky), and $w$ (default). The process evolves as follows:

1. At time $n$, a ball is sampled from the urn. The probability of sampling a particular ball depends on the urn composition after time $n-1$, 
2. According to the color of the sampled ball, the process enters (or remains in) one of the three states of risk. For example, if the sampled ball is of type $x$, the process is in a safe state, whereas it fails if the chosen ball is $w$,

3. The urn is then reinforced according to its reinforcement matrix RM (balanced and constant over time). It means that if the sampled ball is of type $x$, then (it is replaced and) $\theta x$-balls are added to the urn; if the sampled ball is of type $y$, then $\delta y$-balls, and $\lambda z$-balls are added, and if the sampled ball is of type $z$, then $\theta z$-balls are added.

$$
\mathrm{RM}=\begin{gathered}
x \\
w
\end{gathered}\left[\begin{array}{ccc}
1+\theta & y & \\
0 & 1+\delta & \lambda \\
0 & 0 & 1+\theta
\end{array}\right],
$$

where $\lambda=\theta-\delta$.

The distribution and the main properties of the urn process can be described analytically through the analysis of its generating function ${ }^{2}$; see the details in Cirillo and Hüsler [2]. In particular, we can state the following theorem.

THEOREM 4 (Cirillo and Hüsler [1]): Let $X_{n}, Y_{n}$, and $W_{n}$ represent the number of $x$-, $y$-, and $w$-balls in the urn at time $n$. Their moments show to be hypergeometric functions (i.e., finite linear combinations of products and quotients of Euler Gamma functions). In particular, the moments of order l are given by

$$
\begin{aligned}
& E\left[\left(X_{n}\right)_{l}\right]=\theta^{l} \frac{\left(a_{0} / \theta\right)^{(l)}}{\left(t_{0} / \theta\right)^{(l)}} n^{l}+O\left(n^{l-1}\right), \\
& E\left[\left(Y_{n}\right)_{l}\right]=\delta^{l} \frac{\left(b_{0} / \delta\right)^{(l)}}{\left(t_{0} / \theta\right)^{(l \delta / \theta)}} n^{l \delta / \theta}+O\left(n^{(l-1) \delta / \theta}\right), \\
& E\left[\left(W_{n}\right)_{l}\right]=\lambda^{l} \frac{\left(t_{0}-a_{0} / \theta\right)^{(l)}}{\left(t_{0} / \theta\right)^{(l \lambda / \theta)}} n^{l \delta / \theta}+O\left(n^{(l-1) \delta / \theta}\right),
\end{aligned}
$$

where $t_{0}=a_{0}+b_{0}+c_{0}, \lambda=\theta-\delta$, and $(\cdot)^{(n)}=\Gamma(x+n) / \Gamma(x)$ represents the standard Pochhammer formula.

Other results about the limit law of $X_{n}, Y_{n}$, and $W_{n}$ can be found in Cirillo and Hüsler [1], together with results about the asymptotic exchangeability of the triangular urn process and its use from a Bayesian point of view.

The UbGESM is shown to be very flexible and it is able to indirectly reproduce all the main results of Gut and Hüsler [9]. For example, computing the probability

\footnotetext{
${ }^{2}$ The generating function of urn histories (gfuhs) is a generating function of the form $H(z ; x, y, w)=$ $\sum_{n=0}^{\infty} f_{n}(x, y, w)\left(z^{n} / n !\right)$, where $f_{n}(x, y, w)$ is a counting function that counts the number of $x$-, $y$-, and $w$-balls in sampling sequences of length $n$. The gfuh is then that generating function which enumerates all the possible compositions of the urn at time $n$, given its reinforcement matrix and initial composition.
} 
that $Y_{10}=b_{0}+1$ is like asking which is the probability for the system to overcome the risky threshold for the first time in $n=10$. In the same way, $P\left[W_{n}=c_{0}+1\right]$ represents the probability for the model to fail at time $n$.

To model the possibly increasing threshold using the urn-based approach, we need to introduce a four-color $(x, u, y, w)$ urn, with initial composition $\left(a_{0}, d_{0}, b_{0}, c_{0}\right)$. A possible reinforcement matrix ${ }^{3}$ can be the following:

$$
\mathrm{RM}_{2}=\begin{gathered}
x \\
u \\
y \\
w
\end{gathered}\left[\begin{array}{ccccc}
1+\theta & 0 & 0 & 0 \\
\theta & 1 & 0 & 0 \\
0 & 0 & 1+\delta & \lambda \\
0 & 0 & 0 & 1+\theta
\end{array}\right]
$$

This matrix tells us that the balls of color $x, y$, and $w$ behave as in the standard UbGESM, whereas balls of color $u$ represent the strengthening shock. In fact, every time a $u$-ball is sampled, the ball is not replaced in the urn and $\theta x$-balls are added instead. For what concerns the process, it remains in the state it is actually visiting. In this way, some strengthening shocks might increase the probability of entering or remaining in the safe state, indirectly reproducing the increasing threshold mechanism for running-in. If we want all of the $u$-balls to be removed sooner or later, in order to avoid further strengthening shocks, we can, for example modify the second row of the $\mathrm{RM}_{2}$ matrix by a changing $\theta$ with $\theta+1$ and 1 with 0 .

THEOREM 5: Consider an urn process characterized by the reinforcement matrix $R M_{2}$ and with an initial composition $\left(a_{0}, d_{0}, b_{0}, c_{0}\right)$ of balls. The five-variable gfuhs is:

$$
\begin{aligned}
H(z ; x, u, y, w)= & x^{a_{0}} u^{d_{0}} y^{b_{0}} w^{c_{0}}\left(1-\theta x^{\theta} u z\right)^{-\left(a_{0}+d_{0} / \theta\right)}\left(1-\theta w^{\theta} z\right)^{-c_{0} / \theta} \\
& \times\left(1-y^{\delta} w^{-\delta}\left(1-\left(1-\theta w^{\theta} z\right)^{\frac{\delta}{\theta}}\right)\right)^{-b_{0} / \delta}
\end{aligned}
$$

ProOF: First, notice that states $x$ and $u$ are not directly dependent on states $y$ and $w$ through reinforcement and vice versa. Hence matrix $\mathrm{RM}_{2}$ can be seen as a combination of a records urn (Flajolet et al. [4]) for $x$ and $u$ and a triangular urn for $y$ and $w$. The result then comes from a direct application of the isomorphism theorem of Flajolet, Gabarro, and Pekari [5].

Given the gfuhs, we can then study the evolution of the balls in the urn as in Cirillo and Hüsler [1].

\footnotetext{
${ }^{3}$ We could also think of a sacrificial urn (Flajolet, Dumas, and Puyhaubert [4]), in which $w$-balls are removed every time a $u$-ball is sampled.
} 
THEOREM 6: Let $X_{n}, U_{n}, Y_{n}$, and $W_{n}$ represent the number of $x-, u-, y$-, and $w$-balls in the urn at time $n$. Their moments of order l are given by

$$
\begin{aligned}
& E\left[\left(X_{n}\right)_{l}\right]=\theta^{l} \frac{\left(a_{0}+d_{0} / \theta\right)^{(l)}}{\left(t_{0} / \theta\right)^{(l)}} n^{l}+O\left(n^{l-1}\right), \\
& E\left[\left(U_{n}\right)_{l}\right]=d_{0} \\
& E\left[\left(Y_{n}\right)_{l}\right]=\delta^{l} \frac{\left(b_{0} / \delta\right)^{(l)}}{\left(t_{0} / \theta\right)^{(l \delta / \theta)}} n^{l \delta / \theta}+O\left(n^{(l-1) \delta / \theta}\right), \\
& E\left[\left(W_{n}\right)_{l}\right]=\lambda^{l} \frac{\left(\left(t_{0}-a_{0} / \theta\right)\right)^{(l)}}{\left(t_{0} / \theta\right)^{(l \lambda / \theta)}} n^{l \delta / \theta}+O\left(n^{(l-1) \delta / \theta}\right),
\end{aligned}
$$

where $t_{0}=a_{0}+b_{0}+c_{0}+d_{0}$ and $\lambda=\theta-\delta$.

PROOF: Matrix $\mathrm{RM}_{2}$ can be seen as a combination of a records urn for $x$ and $u$ and a triangular urn for $y$ and $w$. In particular, notice that the number of $u$-balls does not vary over time. For what concerns $X_{n}, Y_{n}$, and $W_{n}$, we only show the proof for $y$-balls, as the methodology is always the same. Set $C_{n}=\Gamma(n+1) /\left(t_{0} / \theta\right)^{(n)}$. Taking derivatives of the multivariate generating function, one has

$$
E\left[\left(Y_{n}\right)_{l}\right]=E\left[Y_{n}\left(Y_{n}-1\right) \cdots\left(Y_{n}-l+1\right)\right]=\left.C_{n}\left[z^{n}\right] \frac{\partial^{l} H}{\partial y^{l}}\right|_{x=1, w=1},
$$

where $\left[z^{n}\right]$ represents the standard notation for the operation of coefficient extraction (let $f(X)=\sum_{i=0}^{\infty} u_{i} z^{i}$ be a generating function, then $\left.\left[z^{m}\right] f(z)=\left[z^{m}\right] \sum_{i=0}^{\infty} a_{i} z^{i}=a_{m}\right)$.

With some simple manipulations, we have that

$$
E\left[\left(Y_{n}\right)_{l}\right]=C_{n}\left[\left(\frac{b_{0}}{\delta}\right)^{(l)}(1-\theta z)^{-\left(t_{0}+l \theta\right) \delta / \theta}+\left(\frac{b_{1}}{\delta}\right)^{(l)}(1-\theta z)^{-\left(t_{0}+(l-1) \theta\right) \delta / \theta}+\cdots\right] .
$$

At this point, noting that for $\gamma_{1}<\gamma_{2},\left[z^{n}\right](1-z)^{-\gamma_{1}}=o\left(\left[z^{n}\right](1-z)^{-\gamma_{2}}\right)$, we discover that only the first term influences the asymptotic behavior. So, thanks to a coefficient extraction with respect to $z$, we get the desired result.

At this point, as shown in Flajolet et al. [4] or in Cirillo and Hüsler [1], one can study all of the other properties of the urn process. Such a study goes beyond the scope of the present section, whose aim is simply to build a bridge between generalized shock models with increasing threshold and urn-based shock models. It goes without saying that several different urn processes can be used to develop the alternative modeling.

\section{CONCLUSION}

We have proposed an extension of the GESM by introducing a possibly increasing failure threshold. Although standard models assume that the crucial threshold for the 
system might only decrease over time, because of weakening shocks and obsolescence, we have assumed that, in particular at the beginning of the system's life, some strengthening shocks might increase the system tolerance to large shock, as it happens in running-in phases. This is, for example, the case of turbines' breaking-in in the field of engineering. However, other fields of applications are easily identifiable. On the basis of parametric assumptions, we have provided theoretical results and derived some exact and asymptotic univariate and multivariate distributions for the model.

In the last part of the article we have also shown how to link the new model to some recent urn-based nonparametric approaches proposed in the literature [1].

\section{Acknowledgement}

The present work has been supported by the Swiss National Science Foundation.

\section{References}

1. Cirillo, P. \& Hüsler, J. (2009). An urn approach to generalized extreme shock models. Statistics and Probability Letters 79: 969-976.

2. Cirillo, P. \& Hüsler, J. (2010). Extreme shock models: an alternative perspective. Statistics and Probability Letters 81: 25-30.

3. Dunn, C.W., Pugh, P.R., \& Haddock, S.H.D. (2005). Molecular phylogenetics of the Siphonophora (Cnidaria), with implications for the evolution of functional specialization. Systematic Biology 54: 916-935.

4. Flajolet, P., Dumas, P., \& Puyhaubert, V. (2006). Some exactly solvable models of urn process theory. In Fourth Colloquium on Mathematics and Computer Science, pp. 59-118.

5. Flajolet, P., Gabarro, J., \& Pekari, H. (2005). Analytic urns. Annals of Probability 33: 1200-1233.

6. Gut, A. (1990). Cumulative shock models. Advances in Applied Probability 22: 504-507.

7. Gut, A. (2001). Mixed shock models. Bernoulli 7: 541-555.

8. Gut, A. \& Hüsler, J. (1999). Extreme shock models. Extremes 2: 293-305.

9. Gut, A. \& Hüsler, J. (2005). Realistic variation of shock models. Statistics and Probability Letters 74: 187-204.

10. Muliere, P., Secchi, P., \& Walker, S. (2000). Urn schemes and reinforced random walks. Stochastic Processes and their Applications 88: 59-78. 